\title{
GENERALIZATION OF CERTAIN SUBCLASSES OF ANALYTIC FUNCTIONS
}

\author{
TADAYUKI SEKINE \\ Department of Mathemetics \\ Science and Technology \\ Nihon University \\ 1-8 Kanda Surugadai, Chi yoda-ku \\ Tokyo 101, Japan
}

(Received June 16, 1986 and in revised form September 18, 1986)

ABSTRACT. We introduce the subclass $T_{j}(n, m, \alpha)$ of analytic functions with negative coefficients by the operator $D^{n}$. Coefficient inequalities and distortion theorems of functions in $T_{j}(n, m, \alpha)$ are determind. Further, distortion theorems for fractional calculus of functions in $T_{j}(n, m, \alpha)$ are obtained.

KEYWORDS AND PHRASES. Analytic functions, negative coefficients, coefficient inequalities, distortion theorem, fractional calculus.

1980 AMS SUBJECT CLASSIFICATION CODES. Primary 30C45, Secondary 26 A24.

\section{INTRODUCTION.}

Let $\boldsymbol{A}_{j}$ denote the class of functions of the form

$$
f(z)=z+\sum_{k=j+1}^{\infty} a_{k} z^{k} \quad(j \in N=\{1,2,3, \ldots\})
$$

which are analytic in the unit disk $U=\{z:|z|<1\}$.

For a function $f(z)$ in $A_{j}$, we define

$$
\begin{gathered}
D^{0} f(z)=f(z), \\
D^{1} f(z)=D f(z)=z f^{\prime}(z),
\end{gathered}
$$

and

$$
D^{n} f(z)=D\left(D^{n-1} f(z)\right) \quad(n \in N)
$$

With the above operator $D^{n}$, we say that a function $f(z)$ belonging to $A_{j}$ is in the class $A_{j}(n, m, \alpha)$ if and only if

$$
\operatorname{Re}\left(\frac{D^{n+m} f(z)}{D^{n} f(z)}\right)>\alpha \quad\left(n, m \in N_{0}=N \cup\{0\}\right)
$$

for some $\alpha(0 \leqq \alpha<1)$, and for a11 $z \in U$. 
We note that $A_{1}(0,1, \alpha)=S^{*}(\alpha)$ is the class of starlike functions of order $\alpha$, $A_{1}(1,1, \alpha)=\mathbf{K}(\alpha)$ is the class of convex functions of order $\alpha$, and that $A_{1}(n, 1, \alpha)$ $=S_{n}(\alpha)$ is the class of functions defined by Salagean [1].

Let $T_{j}$ denote the subclass of $A_{j}$ consisting of functions of the form

$$
f(z)=z-\sum_{k=j+1}^{\infty} a_{k} z^{k} \quad\left(a_{k} \geqq 0 ; j \in N\right) \text {. }
$$

Further, we define the class $T_{j}(n, m, \alpha)$ by

$$
T_{j}(n, m, \alpha)=A_{j}(n, m, \alpha) \cap T_{j}
$$

Then we observe that $T_{1}(0,1, \alpha)=T^{*}(\alpha)$ is the subclass of starlike functions of order $\alpha$ (Silverman [2]), $\mathbf{T}_{1}(1,1, \alpha)=\mathbf{C}(\alpha)$ is the subclass of convex functions of order $\alpha$ (Silverman [2]), and that $T_{j}(0,1, \alpha)$ and $T_{j}(1,1, \alpha)$ are the classes defined by Chatterjea [3].

\section{DISTORTION THEOREMS.}

We begin with the statement and the proof of the following result.

LaMA 1. Let the function $f(z)$ be defined by (1.6) with $j=1$. Then $f(z) \in T_{1}(n, m, \alpha)$ if and only if

$$
\sum_{k=2}^{\infty} k^{n}\left(k^{m}-\alpha\right) a_{k} \leqq 1-\alpha
$$

for $\mathrm{n} \in \mathrm{N}_{0}, \mathrm{~m} \in \mathrm{N}_{0}$, and $0 \leqq \alpha<1$. The result is sharp.

PROOF. Assume that the inequality (2.1) holds and let $|z|=1$. Then we have

$$
\begin{aligned}
\left|\frac{D^{n+m_{f}} f(z)}{D^{n} f(z)}-1\right| \leqq & \frac{\sum_{k=2}^{\infty} k^{n}\left(k^{m}-1\right) a_{k}|z|^{k-1}}{1-\sum_{k=2}^{\infty} k^{n} a_{k}|z|^{k-1}} \\
& =\frac{\sum_{k=2}^{\infty} k^{n}\left(k^{m}-1\right) a_{k}}{1-\sum_{k=2}^{\infty} k^{n} a_{k}} \\
& \leqq 1-\alpha
\end{aligned}
$$

which implies (1.5). Thus it follows from this fact that $f(z) \in T_{1}(n, m, \alpha)$.

Conversely, assume that the function $f(z)$ is in the class $T_{1}(n, m, \alpha)$. Then

$$
\operatorname{Re}\left(\frac{D^{n+m} f(z)}{D^{n} f(z)}\right)=\operatorname{Re}\left(\frac{1-\sum_{k=2}^{\infty} k^{n+m} a_{k} z^{k-1}}{1-\sum_{k=2}^{\infty} k^{n} a_{k} z^{k-1}}\right)
$$


for $z \in U$. Choose values of $z$ on the real axis so that $D^{n+m} f(z) / D^{n} f(z)$ is real. Upon clearing the denominator in (2.3) and letting $z \rightarrow 1^{-}$through real values, we obtain

$$
1-\sum_{k=2}^{\infty} k^{n+m} a_{k} \geqq \alpha\left(1-\sum_{k=2}^{\infty} k^{n} a_{k}\right)
$$

which gives (2.1). The result is sharp with the extremal function $f(z)$ defined by

$$
f(z)=z-\frac{1-\alpha}{k^{n}\left(k^{m}-\alpha\right)} z^{k} \quad(k \geqq 2)
$$

REMARK 1. In view of Lemma $1, \mathrm{~T}_{1}(\mathrm{n}, \mathrm{m}, \alpha)$ when $n \in N_{0}$ and $m \in N$ is the subclass of $\mathrm{T}^{*}(\alpha)$ introduced by Silverman [2], and $\mathrm{T}_{1}(\mathrm{n}, \mathrm{m}, \alpha)$ when $\mathrm{n} \in \mathrm{N}$ and $\mathrm{m} \in \mathbf{N}$ is the subclass of $\mathrm{C}(\alpha)$ introduced by Silverman [2].

With the aid of Lemma 1 , we prove

THEOREM 1. Let the function $f(z)$ be defined by (1.6). Then $f(z) \in T_{j}(n, m, \alpha)$ if and only if

$$
\sum_{k=j+1}^{\infty} k^{n}\left(k^{m}-\alpha\right) a_{k} \leq 1-\alpha
$$

for $\mathrm{n} \in \mathrm{N}_{0}, \mathrm{~m} \in \mathrm{N}_{0}$ and $0 \leqq \alpha<1$. The result is sharp for the function

$$
f(z)=z-\frac{1-\alpha}{k^{n}\left(k^{m}-\alpha\right)} z^{k} \quad(k \geqq j+1) .
$$

PROOF. Putting $a_{k}=0(k=2,3,4, \ldots, j)$ in Lemma 1 , we can prove the assertion of Theorem 1 .

COROLLARY 1. Let the function $f(z)$ defined by $(1.6)$ be in the class $T_{j}(n, m, \alpha)$. Then

$$
a_{k} \leqq \frac{1-\alpha}{k^{n}\left(k^{m}-\alpha\right)} \quad(k \geqq j+1) .
$$

The equality in (2.8) is attained for the function $f(z)$ given by (2.7).

COROLLARY 2. $T_{j}(n+1, m, \alpha) \subset T_{j}(n, m, \alpha)$ and $T_{j}(n, m+1, \alpha) \subset T_{j}(n, m, \alpha)$.

REMARK 2, Taking $(j, n, m)=(1,0,1)$ and $(j, n, m)=(1,1,1)$ in Theorem 1 , we have the corresponding results by Silverman [2]. Taking $(j, n, m)=(j, 0,1)$ and $(j, n, m)$ $=(1,1,1)$ in Theorem 1 , we have the corresponding results by Chatter jea [3].

THEOREM 2. Let the function $f(z)$ defined by $(1.6)$ be in the class $T_{j}(n, m, \alpha)$. Then

$$
\left|D^{i} f(z)\right| \geqq|z|-\frac{1-\alpha}{(j+1)^{n-i}\left\{(j+1)^{m}-\alpha\right\}}|z|^{j+1}
$$

and

$$
\left|D^{i} f(z)\right| \leqq|z|+\frac{1-\alpha}{(j+1)^{n-i}\left\{(j+1)^{m}-\alpha\right\}}|z|^{j+1}
$$

for $z \in U$, where $0 \leqq i \leqq n$. The equalities in (2.9) and (2.10) are attained for the 
function $f(z)$ given by

$$
f(z)=z-\frac{1-\alpha}{(j+1)^{n}\left\{(j+1)^{m}-\alpha\right\}} z^{j+1}
$$

PROOF. Note that $f(z) \in T_{j}(n, m, \alpha)$ if and only if $D^{i} f(z) \in T_{j}(n-i, m, \alpha)$, and that

$$
D^{i} f(z)=z-\sum_{k=j+1}^{\infty} k^{i} a_{k} z^{k}
$$

Using Theorem 1, we know that

$$
(j+1)^{n-i}\left\{(j+1)^{m}-\alpha\right\} \sum_{k=j+1}^{\infty} k^{i} a_{k} \leqq 1-\alpha,
$$

that is, that

$$
\sum_{k=j+1}^{\infty} k^{i} a_{k} \leqq \frac{1-\alpha}{(j+1)^{n-i}\left\{(j+1)^{m}-\alpha\right\}} .
$$

It follows from $(2.12)$ and $(2.14)$ that

$$
\left|D^{i} f(z)\right| \geqq|z|-\frac{1-\alpha}{(j+1)^{n-i}\left\{(j+1)^{m}-\alpha\right\}}|z|^{j+1}
$$

and

$$
\left|D^{i} f(z)\right| \leqq|z|+\frac{1-\alpha}{(j+1)^{n-i}\left\{(j+1)^{m}-\alpha\right\}}|z|^{j+1} .
$$

Finally, we note that the equalities in (2.9) and (2.10) are attained for the function $f(z)$ defined by

$$
D^{i} f(z)=z-\frac{1-\alpha}{(j+1)^{n-i}\left\{(j+1)^{m}-\alpha\right\}} z^{j+1} .
$$

This completes the proof of Theorem 2 .

COROLlarY 3. Let the function $f(z)$ defined by $(1.6)$ be in the class $T_{j}(n, m, \alpha)$. Then

$$
|f(z)| \geqq|z|-\frac{1-\alpha}{(j+1)^{n}\left\{(j+1)^{m}-\alpha\right\}}|z|^{j+1}
$$

and

$$
|f(z)| \leqq|z|+\frac{1-\alpha}{(j+1)^{n}\left\{(j+1)^{m}-\alpha\right\}}|z|^{j+1}
$$

for $z \in U$. The equalities in (2.18) and (2.19) are attained for the function $f(z)$ given by $(2.11)$.

PROOF. Taking $i=0$ in Theorem 2, we can easily show (2.18) and (2.19). 
COROLLaRY 4. Let the function $f(z)$ defined by $(1.6)$ be in the class $T_{j}(n, m, \alpha)$. Then

$$
\left|f^{\prime}(z)\right| \geqq 1-\frac{1-\alpha}{(j+1)^{n-1}\left\{(j+1)^{m}-\alpha\right\}}|z|^{j}
$$

and

$$
\left|f^{\prime}(z)\right| \leqq 1+\frac{1-\alpha}{(j+1)^{n-1}\left\{(j+1)^{m}-\alpha\right\}}|z|^{j}
$$

for $z \in U$. The equalities in (2.20) and (2.21) are attained for the function $f(z)$ given by $(2.11)$.

PROOF. Note that $\operatorname{Df}(z)=z f^{\prime}(z)$. Hence, making $i=1$ in Thorem 2, we have the corollary.

REMARK 3. Taking $(j, n, m)=(1,0,1)$ and $(j, n, m)=(1,1,1)$ in Corollary 3 and Corollary 4, we have distortion theorems due to Silverman [2].

\section{DISTORTION THEOREMS FOR FRACTIONAL CALCULUS.}

In this section, we use the following definitions of fractional calculus by Owa [4].

DEFINITION 1. The fractional integral of order $\lambda$ is defined by

$$
D_{z}^{-\lambda} f(z)=\frac{1}{\Gamma(\lambda)} \int_{0}^{z} \frac{f(z)}{(z-\xi)^{1-\lambda}} d \xi
$$

where $\lambda>0, f(z)$ is an analytic function in a simply connected region of the $z$-plane containing the origin and the multiplicity of $(z-\xi)^{\lambda-1}$ is removed by requiring $\log (z-\xi)$ to be real when $(z-\xi)>0$.

DEFINITION 2. The fractional derivative of order $\lambda$ is defined by

$$
D_{z}^{\lambda} f(z)=\frac{1}{\Gamma(1-\lambda)} \frac{d}{d z} \int_{0}^{z} \frac{f(\xi)}{(z-\xi)^{\lambda}} d \xi,
$$

where $0 \leqq \lambda<1, f(z)$ is an analytic function in a simply connected region of the zplane contining the origin and the multiplicity of $(z-\xi)^{-\lambda}$ is removed by requiring $\log (z-\xi)$ to be real when $(z-\xi)>0$.

DEFINITION 3. Under the hypotheses of Definition 2, the fractional derivative of order $(n+\lambda)$ is defined by

$$
D_{z}^{n+\lambda} f(z)=\frac{d^{n}}{d z^{n}} D_{n}^{\lambda} f(z)
$$

where $0 \leqq \lambda<1$ and $n \quad N_{0}=\{0,1,2,3, \ldots\}$.

THEOREM 3. Let the function $f(z)$ defined by $(1.6)$ be in the class $T_{j}(n, m, \alpha)$. Then

$$
\left|D_{z}^{-\lambda}\left(D^{i} f(z)\right)\right| \geq \frac{|z|^{1+\lambda}}{\Gamma(2+\lambda)}\left(1-\frac{\Gamma(j+2) \Gamma(2+\lambda) \cdot(1-\alpha)}{\Gamma(j+2+\lambda)(j+1)^{n-i}\left\{(j+1)^{m}-\alpha\right\}}|z|^{j}\right)
$$


and

$$
\left.\left|D_{z}^{-\lambda}\left(D^{i} f(z)\right)\right| \leqq\left.\frac{|z|^{1+\lambda}}{\Gamma(2+\lambda)}\left|1+\frac{\Gamma(j+2) \Gamma(2+\lambda) \cdot(1-\alpha)}{\Gamma(j+2+\lambda)(j+1)^{n-i}\left\{(j+1)^{m}-\alpha\right\}}\right| z\right|^{j}\right)
$$

for $\lambda>0,0 \leqq i \leqq n$, and $z \quad U$. The equalities in (3.4) and (3.5) are attained for the function $f(z)$ given by (2.11).

PROOF. It is easy to see that

$$
\Gamma(2+\lambda) z^{-\lambda} D_{z}^{-\lambda}\left(D^{i} f(z)\right)=z-\sum_{k=j+1}^{\infty} \frac{\Gamma(k+1) \Gamma(2+\lambda)_{k}{ }^{i} a_{k} z^{k}}{\Gamma(k+1+\lambda)}
$$

Since the function

$$
\phi(k)=\frac{\Gamma(k+1) \Gamma(2+\lambda)}{\Gamma(k+1+\lambda)} \quad(k \geqq j+1)
$$

is decreasing in $k$, we have

$$
0<\phi(k) \leqq \phi(j+1)=\frac{\Gamma(j+2) \Gamma(2+\lambda)}{\Gamma(j+2+\lambda)} .
$$

Therefore, by using (2.14) and (3.8), we can see that

$$
\begin{gathered}
\left|\Gamma(2+\lambda) z^{-\lambda} D_{z}^{-\lambda}\left(D^{i} f(z)\right)\right| \geqq|z|-\phi(j+1)|z|^{j+1} \sum_{k=j+1}^{\infty} k^{i} a_{k} \\
\geqq|z|-\frac{\Gamma(j+2) \Gamma(2+\lambda) \cdot(1-\alpha)}{\Gamma(j+2+\lambda)(j+1)^{n-i}\left\{(j+1)^{m}-\alpha\right\}}|z|^{j+1}
\end{gathered}
$$

which implies (3.4), and that

$$
\begin{gathered}
\left|\Gamma(2+\lambda) z^{-\lambda} D_{z}^{-\lambda}\left(D^{i} f(z)\right)\right| \leqq|z|+\phi(j+1)|z|^{j+1} \sum_{k=j+1}^{\infty} k^{i} a_{k} \\
\leqq|z|+\frac{\Gamma(j+2) \Gamma(2+\lambda) \cdot(1-\alpha)}{\Gamma(j+2+\lambda)(j+1)^{n-i}\left\{(j+1)^{m}-\alpha\right\}}|z|^{j+1}
\end{gathered}
$$

which shows (3.5). Furthermore, note that the equalities in (3.4) and (3.5) are attained for the function $f(z)$ defined by

$$
D_{z}^{-\lambda}\left(D^{i} f(z)\right)=\frac{z^{1+\lambda}}{\Gamma(2+\lambda)}\left(1-\frac{\Gamma(j+2) \Gamma(2+\lambda) \cdot(1-\alpha)}{\Gamma(j+2+\lambda)(j+1)^{n-i}\left\{(j+1)^{m}-\alpha\right\}} z^{j}\right)
$$

or (2.17). Thus we complete the assertion of Theorem 3 .

Taking $i=0$ in Theorem 3, we have

COROLLARY 5. Let the function $f(z)$ by $(1.6)$ be in the class $T_{j}(n, m, \alpha)$. Then 


$$
\left.\left|D_{z}^{-\lambda} f(z)\right| \geqq\left.\frac{|z|^{1+\lambda}}{\Gamma(2+\lambda)}\left|1-\frac{\Gamma(j+2) \Gamma(2+\lambda) \cdot(1-\alpha)}{\Gamma(j+2+\lambda)(j+1)^{n}\left\{(j+1)^{m}-\alpha\right\}}\right| z\right|^{j}\right)
$$

and

$$
\left.\left|D_{z}^{-\lambda} f(z)\right| \leqq\left.\frac{|z|^{1+\lambda}}{\Gamma(2+\lambda)}\left|1+\frac{\Gamma(j+2) \Gamma(2+\lambda) \cdot(1-\alpha)}{\Gamma(j+2+\lambda)(j+1)^{n}\left\{(j+1)^{m}-\alpha\right\}}\right| z\right|^{j}\right)
$$

for $\lambda>0$ and $z \in U$. The equalities in (3.12) and (3.13) are attained for the function $f(z)$ given by (2.11).

Finally, we prove

THBOREM 4. Let the function $f(z)$ defined by $(1.6)$ be in the class $T_{j}(n, m, \alpha)$. Then

$$
\left.\left|D_{z}^{\lambda}\left(D^{i} f(z)\right)\right| \geqq\left.\frac{|z|^{1-\lambda}}{\Gamma(2-\lambda)}\left|1-\frac{\Gamma(j+1) \Gamma(2-\lambda) \cdot(1-\alpha)}{\Gamma(j+2-\lambda)(j+1)^{n-i-1}\left\{(j+1)^{m}-\alpha\right\}}\right| z\right|^{j}\right)
$$

and

$$
\left.\left|D_{z}^{\lambda}\left(D^{i} f(z)\right)\right| \leqq\left.\frac{|z|^{1-\lambda}}{\Gamma(2-\lambda)}\left|1+\frac{\Gamma(j+1) \Gamma(2-\lambda) \cdot(1-\alpha)}{\Gamma(j+2-\lambda)(j+1)^{n-i-1}\left\{(j+1)^{m}-\alpha\right\}}\right| z\right|^{j}\right)
$$

for $0 \leqq \lambda<1,0 \leqq i \leqq n-1$, and $z \in U$.

The equalities in (3.14) and (3.15) are attained for the function $f(z)$ given by (2.11).

PROOF. A simple computation gives that

$$
\Gamma(2-\lambda) z D_{z}^{\lambda}\left(D^{i} f(z)\right)=z-\sum_{k=j+1}^{\infty} \frac{\Gamma(k+1) \Gamma(2-\lambda)_{k}}{\Gamma(k+1-\lambda)} a_{k} z^{k}
$$

Note that the function

$$
\psi(k)=\frac{\Gamma(k) \Gamma(2-\lambda)}{\Gamma(k+1-\lambda)} \quad(k \geqq j+1)
$$

is decreasing in $k$. It follows from this fact that

$$
0<\psi(k) \leqq \psi(j+1)=\frac{\Gamma(j+1) \Gamma(2-\lambda)}{\Gamma(j+2-\lambda)} .
$$

Consequently, with the aid of $(2.14)$ and $(3.18)$, we have

$$
\begin{aligned}
& \left|\Gamma(2-\lambda) z^{\lambda} D_{z}^{\lambda}\left(D^{i} f(z)\right)\right| \geqq|z|-\psi(j+1)|z|^{j+1} \sum_{k=j+1}^{\infty} k^{i+1} a_{k} \\
& \geqq|z|-\frac{\Gamma(j+1) \Gamma(2-\lambda) \cdot(1-\alpha)}{\Gamma(j+2-\lambda)(j+1)^{n-i-1}\left\{(j+1)^{m}-\alpha\right\}}|z|^{j+1}
\end{aligned}
$$

and

$$
\left.\Gamma(2-\lambda) z^{\lambda} D_{z}^{\lambda}\left(D^{i} f(z)\right)|\leqq| z|+\psi(j+1)| z\right|^{j+1} \sum_{k=j+1}^{\infty} k^{i+1} a_{k}
$$




$$
\leqq|z|+\frac{\Gamma(j+1) \Gamma(2-\lambda) \cdot(1-\alpha)}{\Gamma(j+2-\lambda)(j+1)^{n-i-1}\left\{(j+1)^{m}-\alpha\right\}}|z|^{j+1} .
$$

Thus (3.14) and (3.15) follow from (3.19) and (3.20), respectively. Further, since the equalities in (3.19) and (3.20) are attained for the function $f(z)$ defined by

$$
D_{z}^{\lambda}\left(D^{i} f(z)\right)=\frac{z^{1-\lambda}}{\Gamma(2-\lambda)}\left(1-\frac{\Gamma(j+1) \Gamma(2-\lambda) \cdot(1-\alpha)}{\Gamma(j+2-\lambda)(j+1)^{n-i-1}\left\{(j+1)^{m}-\alpha\right\}} z^{j}\right),
$$

that is, by (2.17), this completes the proof of Theorem 4 .

Making $i=0$ in Theorem 4, we have

COROLLARY 6. Let the function $f(z)$ defined by $(1.6)$ ber in the class $T_{j}(n, m, \alpha)$. Then

$$
\left|D_{z}^{\lambda} f(z)\right| \geqq \frac{|z|^{1-\lambda}}{\Gamma(2-\lambda)}\left(1-\frac{\Gamma(j+1) \Gamma(2-\lambda) \cdot(1-\alpha)}{\Gamma(j+2-\lambda)(j+1)^{n-1}\left\{(j+1)^{m}-\alpha\right\}}|z|^{j}\right)
$$

and

$$
\left|D_{z}^{\lambda} f(z)\right| \leqq \frac{|z|^{1-\lambda}}{\Gamma(2-\lambda)}\left(1+\frac{\Gamma(j+1) \Gamma(2-\lambda) \cdot(1-\alpha)}{\Gamma(j+2-\lambda)(j+1)^{n-1}\left\{(j+1)^{m}-\alpha\right\}}|z|^{j}\right)
$$

for $0 \leqq \lambda<1$ and $z \in U$. the equalities in (3.22) and (3.23) are attained for the function $f(z)$ given by (2.11).

ACKNOWLBDGEMENT. The author wishes to thank the referee for his helpfull comments.

\section{REFERENCES}

1. SALAGEAN, G.S. Subclasses of univalent functions, Lecture Notes in Math., 1013 (Springer-Verlag, Berlin Heidelberg New York, 1983) 292-310.

2. SILVERMAN, H. Univalent functions with negative coefficients, Proc. Amer. Math. Soc., 51 (1975) 109-116.

3. CHATTERJEE, S.K. On starlike functions, J. Pure Math., 1 (1981) 23-26.

4. OWA, S. On the distortion theorems, Kyungpook Math. J., 18 (1978) 53-59.

5. DEBNATH, L. and GRUM, W.J. The Fractional Calculus and its Role in the Synthesis of Special Functions Part-I and Part-II, Internat. J. Math. Edu. in Sci. and Tech.(to appear) 1986. 


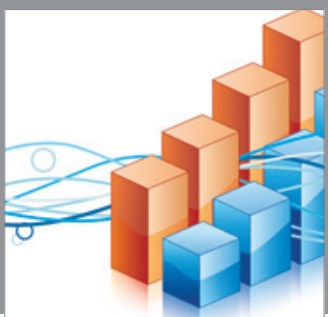

Advances in

Operations Research

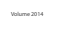

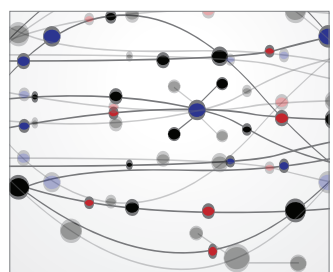

\section{The Scientific} World Journal
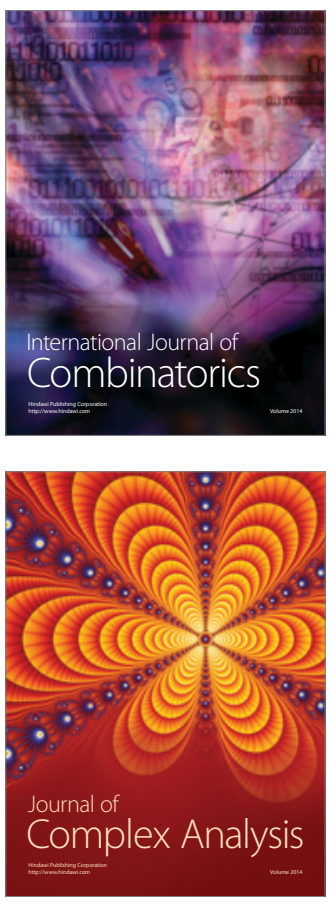

International Journal of

Mathematics and

Mathematical

Sciences
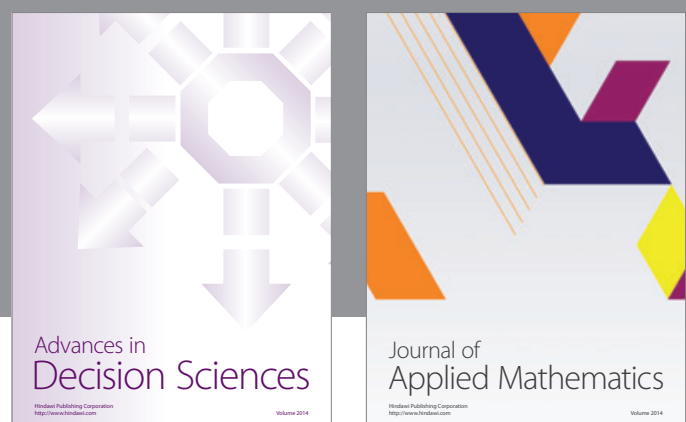

Journal of

Applied Mathematics
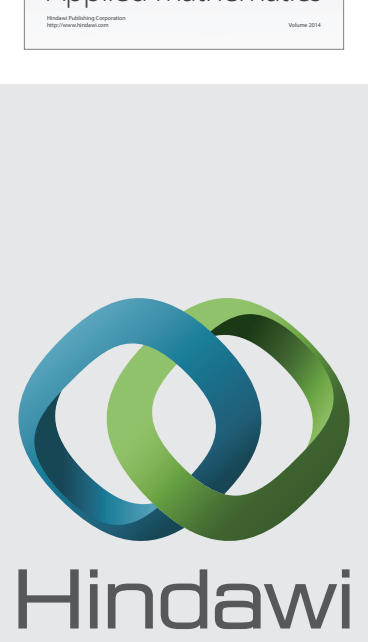

Submit your manuscripts at http://www.hindawi.com
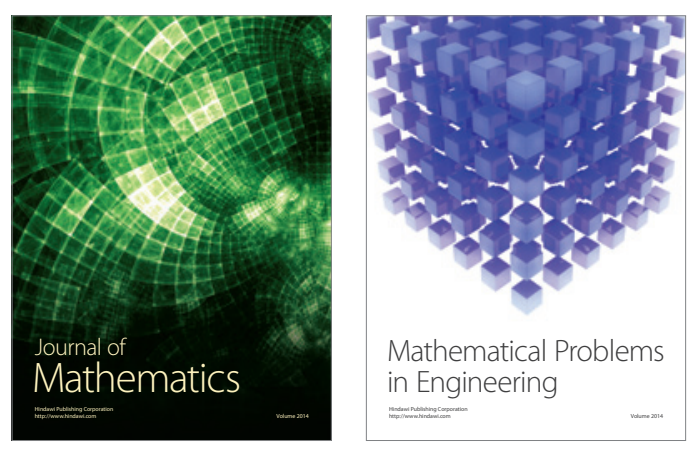

Mathematical Problems in Engineering
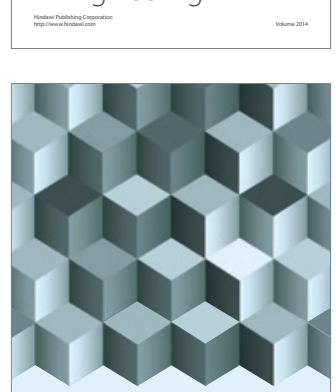

Journal of

Function Spaces
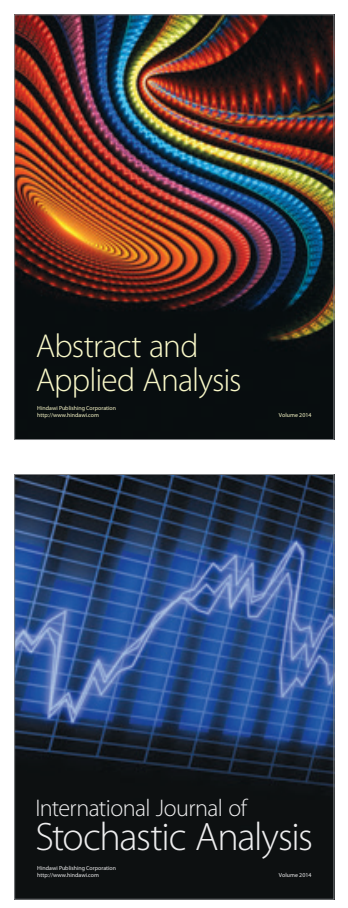

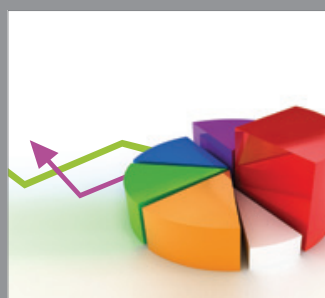

ournal of

Probability and Statistics

Promensencen
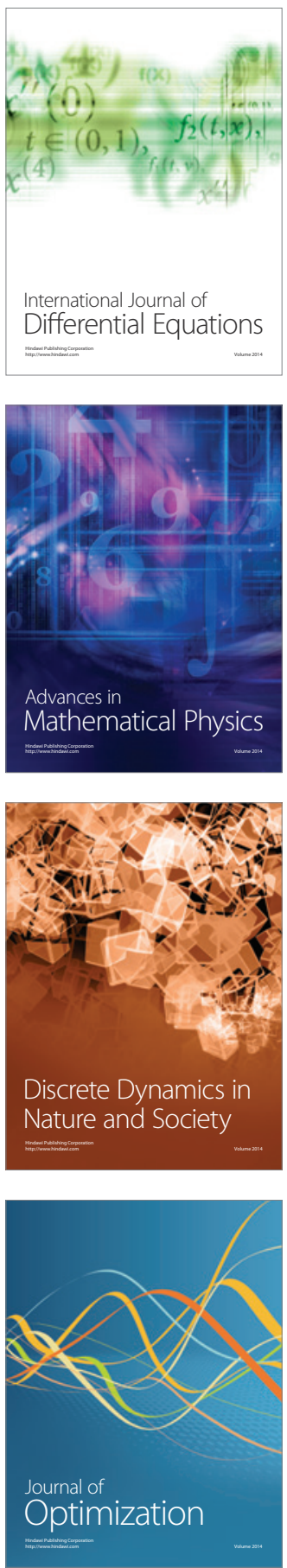